\title{
Polarization instabilities in birefringent fibers: A comparison between continuous waves and solitons
}

\author{
Yijiang Chen and Javid Atai \\ Optical Sciences Centre, Australian National University, Canberra, Australia
}

(Received 10 March 1995)

\begin{abstract}
A comparison between continuous waves (cws) and soliton pulses on the bifurcation character for stationary solutions and polarization instabilities in birefringent fibers is presented. For stationary solutions, solitons are shown to demonstrate qualitatively similar bifurcation character to that of cws. The polarization instabilities for solitons are, however, found to differ from those for cws: the fast soliton is unstable even below the bifurcation point and elliptically polarized solitons are all unstable, in contrast to cws, for which the fast mode is unstable only above the bifurcation point and the elliptically polarized modes are all stable. The reason for the difference is discussed.
\end{abstract}

PACS number(s): 42.81.Dp, 42.81.Gs

In a linear birefringent fiber, the beams with two polarizations propagate independently. At high power, however, two orthogonally polarized beams propagating in a birefringent fiber interact, leading to various fascinating and intriguing nonlinear phenomena for diverse ultrafast optical device applications. For continuous waves (cws), the nonlinearity induced polarization dynamics of light waves in a birefringent fiber (which are applicable to the operation of fiber-optic logic gates and intensity discriminators) have been investigated extensively since 1982 [1-9]. One of the interesting findings reported on the subject is the unstable evolution of the linearly polarized beam initially launched along the fast axis of the birefringent fiber above a critical power [5]. This unstable evolution of the beam along the fast axis consequently results in energy swapping between the two polarizations. The dynamic evolution of nonlinear cws in a birefringent fiber, including the unstable propagation of the beam along the fast axis above the critical power, is in effect equivalent to that of a nonlinear coupler, provided the meaning of physical quantities involved are adequately correlated or the meaning of subscripts in the governing equations for the two systems are properly redefined in transforming the solutions from one system to the other [8]. This fact can be appreciated alternatively by comparing the nonlinear modal or bifurcation diagrams (that portrait the basic character of the system and accordingly reveal dynamic evolutions of nonlinear waves in the system [10]) and the corresponding stability character in the two systems that are qualitatively identical as shown in Ref. [10] for the nonlinear coupler and in Ref. [9] for the birefringent fiber. A light evolution in an untwisted nonlinear birefringent fiber is equivalent to that in a symmetric nonlinear coupler and a twisted birefringent fiber corresponds a mismatched nonlinear coupler $[8,9]$.

Interestingly enough, for solitons it was shown that the nonlinear effects can make the fast mode (soliton) unstable too as in cws when the beat length between the modes is long [11]. To find out whether the propagation of soliton pulse in birefringent fibers does, in general, behave similarly to or differently from that of continuous wave, in the following we present a comparison between the sol- iton and continuous wave propagation in birefringent fibers on their bifurcation and stability characteristics.

The pulse propagation in a birefringent fiber is described by the coupled nonlinear Schrödinger equations $[11,12]$

$$
\begin{aligned}
& i \frac{\partial u}{\partial z}+\frac{1}{2} \frac{\partial^{2} u}{\partial T^{2}}+\kappa u \\
& +\left(|u|^{2}+c|v|^{2}\right) u+(1-c) v^{2} u^{*}=0 \\
& i \frac{\partial v}{\partial z}+\frac{1}{2} \frac{\partial^{2} v}{\partial T^{2}}-\kappa v \\
& +\left(c|u|^{2}+|v|^{2}\right) v+(1-c) u^{2} v^{*}=0
\end{aligned}
$$

where $u$ and $v$ are the normalized envelopes of the slow and fast linearly polarized modes, $\kappa=\left(\beta_{x}-\beta_{y}\right) / 2$ is the linear birefringence that may result from shape or stress, $c$ is cross-phase modulation coefficient that is $\frac{2}{3}$ for silica fibers, and $T$ and $z$ are the normalized time and distance. In terms of circular polarizations $C_{ \pm}=(u \pm i v) /$ $\sqrt{2}$, Eq. (1) reads

$$
\begin{aligned}
i \frac{\partial C_{ \pm}}{\partial z}+\frac{1}{2} \frac{\partial^{2} C_{ \pm}}{\partial T^{2}} & +\kappa C_{\mp} \\
& +\left[c\left|C_{ \pm}\right|^{2}+(2-c)\left|C_{\mp}\right|^{2}\right] C_{ \pm}=0 .
\end{aligned}
$$

These governing equations (1) and (2) for pulses reduce to the ones for the cw when the terms involving the secondorder derivative with respect to $T$ in the equations are dropped.

For cws, the modal or bifurcation diagrams are shown in Fig. 1(a) in terms of total power $\left(P=\left|C_{+}\right|^{2}\right.$ $\left.+\left|C_{-}\right|^{2}=|u|^{2}+|v|^{2}\right)$ vs fraction of power $P_{+}\left(=\left|C_{+}\right|^{2}\right)$ in the right circular polarization for the case of $c=\frac{2}{3}$. The bifurcation diagrams are characterized by pitchfork bifurcation. The modal diagrams in terms of the propagation constant $\beta\left[C_{ \pm}(z)=\left|C_{ \pm}\right| \exp (i \beta z)\right]$ vs the total power $P$ are illustrated in Fig. 1(b). The slow $(u \neq 0, v=0)$ and fast $(u=0, v \neq 0)$ modes exist over the entire range of power. The slow mode is stable for any power whereas the fast mode becomes unstable above a critical power 


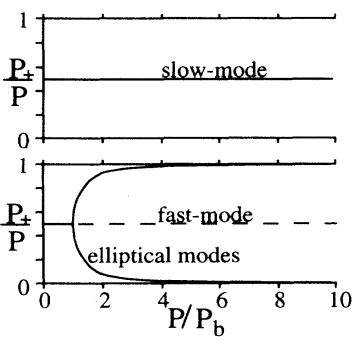

(a)

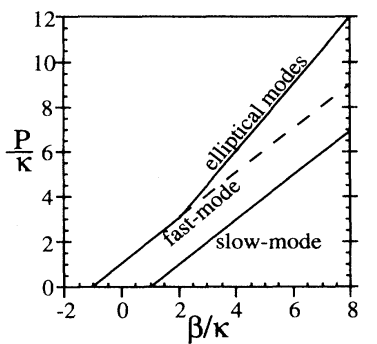

(b)
FIG. 1. Modal or bifurcation diagrams: the relation (a) between the total power $\left(P=\left|C_{+}\right|^{2}+\left|C_{-}\right|^{2}=|u|^{2}+|v|^{2}\right)$ and fraction of power $P_{+}\left(=\left|C_{+}\right|^{2}\right)$ in the right circular polarization and (b) between the propagation constant $\beta$ and the total power $P$ for cws in a birefringent fiber with $c=\frac{2}{3}$.

$P=P_{b}=\kappa /(1-c)=\left(\beta_{x}-\beta_{y}\right) / 2(1-c)$, which also marks the emergence of two stable degenerate elliptically polarized modes when $P>P_{b}[8-10]$. For $c=\frac{2}{3}, P_{b}=3 \kappa$, corresponding to $\beta=\beta_{b}=c \kappa /(1-c)=2 \kappa$.

For pulses, the bifurcation diagrams have qualitatively similar characteristics as shown in Fig. 2 for the case of $c=\frac{2}{3}$, which are obtained by substituting $u(T, z)=f(T) \exp (i \beta z)$ and $v(T, z)=i g(T) \exp (i \beta z)$ into Eq. (1) and solving the resulting equations. The solutions for the slow and fast solitons have simple analytical forms

$$
\left.\begin{array}{c}
u \\
v
\end{array}\right\}=\sqrt{2\left(\beta_{\mp} \kappa\right)} \operatorname{sech}\left[\sqrt{2\left(\beta_{\mp} \kappa\right)} T\right] \exp (i \beta z),
$$

with the corresponding power (or energy of the pulses)

$$
\begin{aligned}
P=\int_{-\infty}^{\infty}\left(|u|^{2}+|v|^{2}\right) d T & =\int_{-\infty}^{\infty}\left(\left|C_{+}\right|^{2}+\left|C_{-}\right|^{2}\right) d T \\
& =2 \sqrt{2\left(\beta_{\mp} \kappa\right)} .
\end{aligned}
$$

For the elliptically polarized beams, the stationary solu-

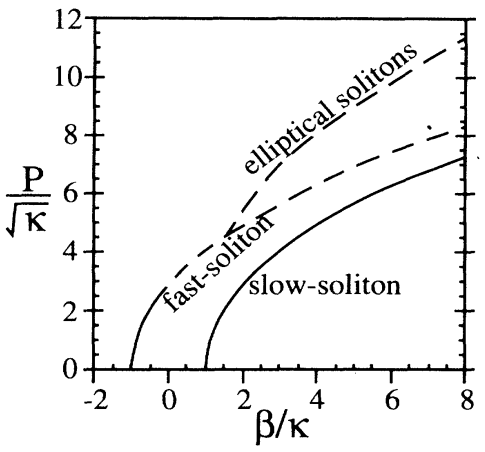

FIG. 2. Modal diagrams in terms of the propagation constant $\beta$ vs the total power (or energy of the pulses) $P$ $\left[=\int_{-\infty}^{\infty}\left(|u|^{2}+|v|^{2}\right) d T=\int_{-\infty}^{\infty}\left(\left|C_{+}\right|^{2}+\left|C_{-}\right|^{2}\right) d T\right]$ for solitons in a birefringent fiber with $c=\frac{2}{3}$. Note that the power $P$ for the solitons presented in longitudinal coordinate is normalized by dividing $\sqrt{\kappa}$ rather than $\kappa$ in the cw case of Fig. 1(b). This is because the physical definition of $P$ for the cw is the sum of intensities of the two orthogonal components, which gives $P$ proportional to $\kappa$, whereas that for the solitons is defined as integral of the sum of intensities of the two orthogonal components over the time, which yields $P$ proportional to $\sqrt{\kappa}$.

tions are derived by solving the coupled equations

$$
\begin{aligned}
& \frac{1}{2} \frac{d^{2} f}{d T^{2}}-(\beta-\kappa) f+\left[f^{2}+(2 c-1) g^{2}\right] f=0 \\
& \frac{1}{2} \frac{d^{2} g}{d T^{2}}-(\beta+\kappa) g+\left[g^{2}+(2 c-1) f^{2}\right] g=0
\end{aligned}
$$

numerically. Although Eq. (4) is not amiable to analytical solutions for the elliptically polarized solitions, the bifurcation point at which the elliptically polarized solitons commence to exist can be derived analytically by a perturbation method [13]. These solitons come into existence when the propagation constant

$$
\beta>\beta_{b}=\kappa[3+4(2 c-1)-\sqrt{8(2 c-1)+1}] /[1-4(2 c-1)+\sqrt{8(2 c-1)+1}],
$$

which is $\beta_{b}=1.529 \kappa$ for $c=\frac{2}{3}$. The associated bifurcation power $P_{b}=2 \sqrt{2(\beta+\kappa)}=4.5 \sqrt{\kappa}$.

Qualitatively, the bifurcation diagrams of Fig. 2 for solitons bear resemblance to those for cws in Fig. 1(b). The stability characteristics for the two cases, however, differ significantly. To reveal the stability feature of the soliton states of Fig. 2, we solve the linearized equation (2)

$$
\begin{aligned}
& i \frac{\partial \delta C_{ \pm}}{\partial z}+\frac{1}{2} \frac{\partial^{2} \delta C_{ \pm}}{\partial T^{2}}+\beta \delta C_{ \pm}+\kappa \delta C_{\mp} \\
& \quad+\left[2 c\left|C_{ \pm 0}\right|^{2}+(2-c)\left|C_{\mp 0}\right|^{2}\right] \delta C_{ \pm} \\
& \quad+c C_{ \pm 0}^{2} \delta C_{ \pm}^{*} \\
& \quad+(2-c) C_{ \pm 0}\left(C_{\mp 0}^{*} \delta C_{\mp}+C_{\mp 0} \delta C_{\mp}^{*}\right)=0,
\end{aligned}
$$

which is obtained by substituting $C_{ \pm}=\left(C_{ \pm 0}\right.$ $\left.+\delta C_{ \pm}\right) \exp (i \beta z)$ into Eq. (2) with $C_{ \pm 0}$ the stationary solutions. The solutions to Eq. (5) for the soliton states of Fig. 2 yield the growth rates $\Delta\left[\delta C_{ \pm}(T, z)\right.$ $\left.=\delta C_{ \pm}(T) \exp (\Delta \kappa z)\right]$ for the elliptically polarized solitons and for the fast soliton with $\beta>0$, but no growth rate for the slow soliton. This means that the slow soliton is stable, the elliptically polarized solitons are unstable, and the fast soliton is unstable above $\beta=0$, which is smaller than the bifurcation value $\beta_{b}=1.529 \kappa$. These stable characteristics for solitons contrast strikingly with cws for which the elliptically polarized modes are stable and the fast mode becomes unstable only above the bifurcation point $\beta=\beta_{b}$ and $P=P_{b}$. Figure 3 illustrates the growth rates for the fast soliton and the elliptically polarized solitons. Above the bifurcation point $\beta>\beta_{b}$ $\left(P>P_{b}\right)$, the instability of the fast soliton results from the existence of the real growth rate ( $\Delta$ being real), whereas 


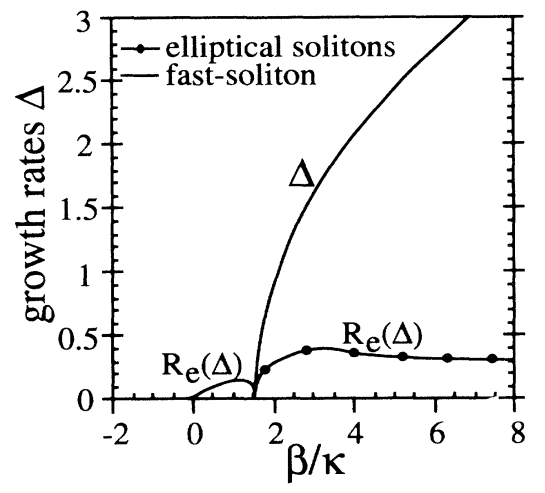

FIG. 3. Growth rates for the fast soliton and the elliptically polarized solitons of Fig. 2.

below $\beta<\beta_{b}\left(P<P_{b}\right)$ the growth rate becomes complex with $\operatorname{Re}(\Delta)$, the real part of $\Delta$, demonstrated in Fig. 3 . On the other hand, the instability of the elliptically polarized solitons results solely from the existence of the complex growth rates $\Delta$ with $\operatorname{Re}(\Delta)$ illustrated in Fig. 3 . These conclusions of the stability for the solitons derived from the linearized equation (5) are consistent with those from directly solving Eq. (1) or (2). Shown in Fig. 4 is the unstable evolution of the elliptically polarized stationary solutions at $\beta / \kappa=3$, which breaks down with the propagation distance around $Z=\kappa z=30$.

The elliptically polarized solitons and the fast soliton are unstable. This instability originates from the power of the modes or the order of the solitons. The slow soliton has the smallest power and it is the fundamental state, whereas the fast soliton and the elliptically polarized solitons carry power higher than the fundamental one and thus are referred to as the higher-order soliton states that can be unstable. In a nonlinear optical system with $(1+1)-D$, the fundamental soliton is normally stable whereas higher-order solitons can be unstable. Equation (4) in fact supports other higher-order solutions with multiple peaks in the intensity profile that have no counterpart in cws [13-15]. These higher-order solitons are unstable too. This unstable nature of higher-order solitons in a birefringent fiber is similar to that in other nonlinear optical systems such as those governed by the single nonlinear Schrödinger equation where higher-

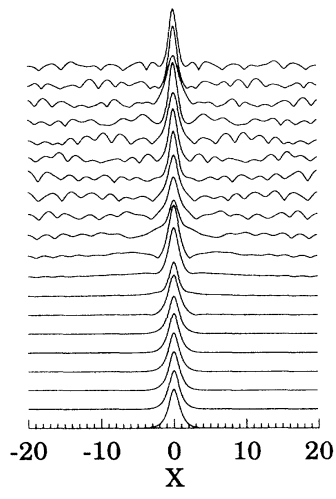

(a)

\section{Z}

100

50

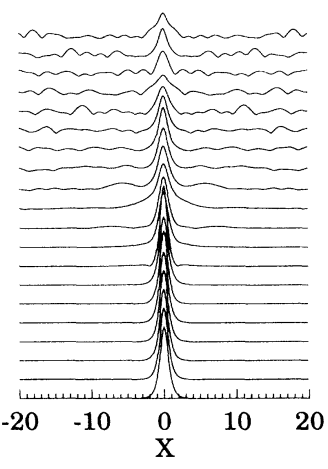

(b)
FIG. 4. Unstable evolution of the elliptically polarized stationary solutions at $\beta / \kappa=3$ of Fig. 2 (a) for $|u| / \sqrt{\kappa}$ and (b) for $|v| / \sqrt{\kappa}$ with $Z=z \kappa$, and $X=T \sqrt{\kappa}$.

order (periodical) solitons are all unstable [16].

Before concluding, it should be added here that for nonlinear couplers, continuous waves and solitons behave similarly in their bifurcation and stability features (and so do their switching and other dynamic characters). This becomes clear if one compares the bifurcation diagram and the corresponding stability for the cws [17] with those for the solitons [18] which demonstrate qualitatively identical characteristics as far as the fundamental state is concerned.

In summary, similarities and differences in the bifurcation character for stationary solutions and polarization instabilities in birefringent fibers between solitons and cws are presented. For stationary solutions, solitons are shown to demonstrate qualitatively similar bifurcation character to that of cws. The polarization instabilities for solitons is, however, found to differ significantly from those for cws: the fast soliton is unstable even below the bifurcation point and elliptically polarized solitons are all unstable, whereas for the cws the fast mode is unstable only above the bifurcation point and the elliptically polarized modes are all stable.

The Optical Sciences Centre is part of the Australian Photonics Cooperative Research Center.
[1] R. H. Stolen, J. Botineau, and A. Ashkin, Opt. Lett. 7, 512 (1982).

[2] B. Nikolaus, D. Grischkowsky, and A. C. Balant, Opt. Lett. 8, 189 (1983).

[3] K. Kitayama, Y. Kimura, and S. Seikai, Appl. Phys. Lett. 46, 317 (1985).

[4] H. G. Winful, Appl. Phys. Lett. 47, 213 (1985).

[5] H. G. Winful, Opt. Lett. 11, 33 (1986).

[6] S. Trillo, S. Wabnitz, R. H. Stolen, G. Assanto, C. T. Seaton, and Stegeman, Appl. Phys. Lett. 49, 1224 (1986); 49, 1206 (1986).

[7] Y. Chen, J. Appl. Phys. 66, 43 (1989).

[8] A. W. Snyder, Y. Chen, D. Rowland, and D. J. Mitchell, Opt. Lett. 15, 171 (1990).
[9] S. F. Feldman, D. A. Weinberger, and H. G. Winful, J. Opt. Soc. Am. B 10, 1191 (1993).

[10] A. W. Snyder, D. J. Mitchell, L. Poladian, D. Rowland, and Y. Chen, Opt. Lett. 15, 171 (1990).

[11] K. J. Blow, N. J. Doran, and D. Wood, Opt. Lett. 12, 202 (1987).

[12] C. R. Menyuk, IEEE J. Quantum Electron. 23, 174 (1987).

[13] V. M. Eleonskii, V. G. Korolev, N. E. Kulagin, and L. P. Shil'nikov, Zh. Eksp. Teor. Fiz. 99, 1113 (1991) [Sov. Phys. JETP 72, 619 (1991)]; N. Akhmediev, A. V. Buryak, J. M. Soto-Crespo, and D. R. Andersen, J. Opt. Soc. Am. B 12, 434 (1995).

[14] M. V. Tratnik and J. E. Sipe. Phys. Rev. A 38, 2011 (1988). 
[15] D. N. Christodoulides and R. I. Joseph, Opt. Lett. 13, 53 (1988).

[16] V. E. Zakarov and A. B. Shabat, Zh. Eksp. Teor. Fiz. 61, 118 (1971) [Sov. Phys. JETP 34, 62 (1972)].

[17] Y. Chen and A. W. Snyder, J. Opt. Soc. Am. B 8, 1621
(1991).

[18] N. Akhmediev and A. Ankiewicz, Phys. Rev. Lett. 70, 2395 (1993); J. M. Soto-Crespo and N. Akhmediev, Phys. Rev. E 48, 4710 (1993). 